\title{
One foot in the door: gene gating supports nuclear export of MYC mRNA
}

OSE-mediated
anchoring of
$M Y C$ to the
nuclear pore
is required
for MYC
transcripts to
escape from
nuclear decay

The tethering of genes to nuclear pores can facilitate reactivation and rapid nuclear export of transcripts, a principle referred to as gene gating. Gene gating has been described in yeast and flies, but its contribution to gene regulation in human cells and to cancer is unknown. Now, Scholz et al. show that gene gating of transcriptionally active $M Y C$ alleles occurs in human colon cancer cells and increases nuclear export and overall expression of $M Y C$ transcripts.

To start with, the researchers constructed networks of chromatin that impact human MYC based on reads generated using the Nodewalk technique, a chromosome conformation capture-based assay to investigate chromatin interactions. To determine the relationship of these networks to nuclear pores, these analyses were combined with genome-wide binding data of nucleoporin 153 (NUP153) and NUP133 (both of which are part of the nuclear pore) in normal human

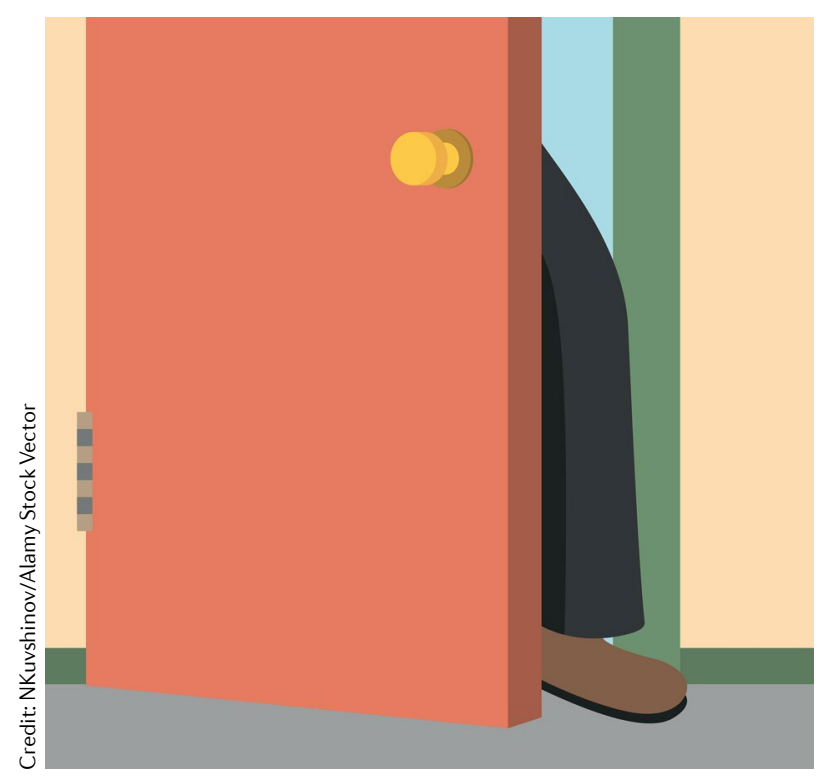

colon epithelial cells (HCECs) and human colon cancer (HCT116) cells. This analysis showed that NUP153 binding was enriched in many of the most highly connected enhancer regions within the two topologically associating domains flanking MYC in both HCECs and HCT116 cells. However, it was not enriched in constitutive laminaassociated domains of peripheral heterochromatin in either cell line. By contrast, in the oncogenic super-enhancer (OSE) region that showed the highest degree of MYC interaction, NUP133 binding was enriched specifically in HCT116 cells, whereas the MYC promoter region in HCT116 cells, and the respective OSE and promoter regions in HCECs, showed little or no NUP133 binding.

Next, the authors explored how the NUP133 and OSE interaction relates to nuclear architecture, using chromatin in situ proximity (ChrISP) assays. ChrISP enables high-resolution, fluorescence-based detection of $<16 \mathrm{~nm}$ physical proximity of NUP133 and digoxygenin-labelled DNA fluorescence in situ hybridization (FISH) probes. HCT116 cells showed higher NUP133-OSE proximity signals than HCECs. Moreover, NUP133-OSE proximity signals in HCT116 cells were higher at the nuclear lamina than in other intranuclear regions, indicating a transient anchoring of OSE to nuclear pores. Analyses of MYCOSE proximities in HCT116 cells revealed that $M Y C-O S E$ proximity signals were highest in the nuclear periphery. By contrast, the proximities of MYC to a MYC enhancer region, EnhD, which is more proximal to $M Y C$ and does not bind NUP153 or NUP133, were lowest in the nuclear periphery.
Could these enhancer proximities be linked to ongoing MYC transcription and differences in nuclear export of nascent $M Y C$ transcripts? RNA-FISH analyses revealed two dominant populations of active MYC alleles, which overlapped with the respective location of enhancer proximities. Importantly, relatively higher levels of transcriptionally active $M Y C$ alleles overlapped with $M Y C-O S E$ proximity signals in the nuclear periphery compared with those overlapping with EnhD-MYC proximity signals.

Analysis of RNA isolated from nuclear or cytosolic fractions from HCT 116 cells and HCECs subjected to a 5-ethynyl uridine pulse-chase to determine nascent MYC transcripts showed that the ratio of cytoplasmic to nuclear $M Y C$ transcripts was higher in HCT116 cells than in HCECs. When inhibiting transcriptional elongation, nuclear MYC transcripts decayed at a higher rate than cytoplasmic transcripts and at similar rates between HCT116 cells and HCECs.

It is known that WNT regulates MYC-specific enhancers via TCF4, and disruption of $\beta$-catenin and TCF 4 complexes by the drug BC21 reduced the interaction between NUP133 and OSE in HCT116 cells. $\mathrm{BC} 21$ reduced the export rate of nascent $M Y C$ transcripts and reduced total cytosolic MYC transcript levels in HCT116 cells, showing that the OSE-mediated anchoring of MYC to the nuclear pore is required for $M Y C$ transcripts to escape from nuclear decay.

This study highlights how nuclear architecture can promote oncogenic gene expression. Whether this mechanism is involved in promoting tumour growth in vivo and in other models of cancer remains to be shown.

Ulrike Harjes

ORIGINAL ARTICLE Scholz, B. A. et al. WNT signaling and AHCTF1 promote oncogenic MYC expression through super-enhancer-mediated gene gating. Nat. Genet. 51, 1723-1731 (2019) 